
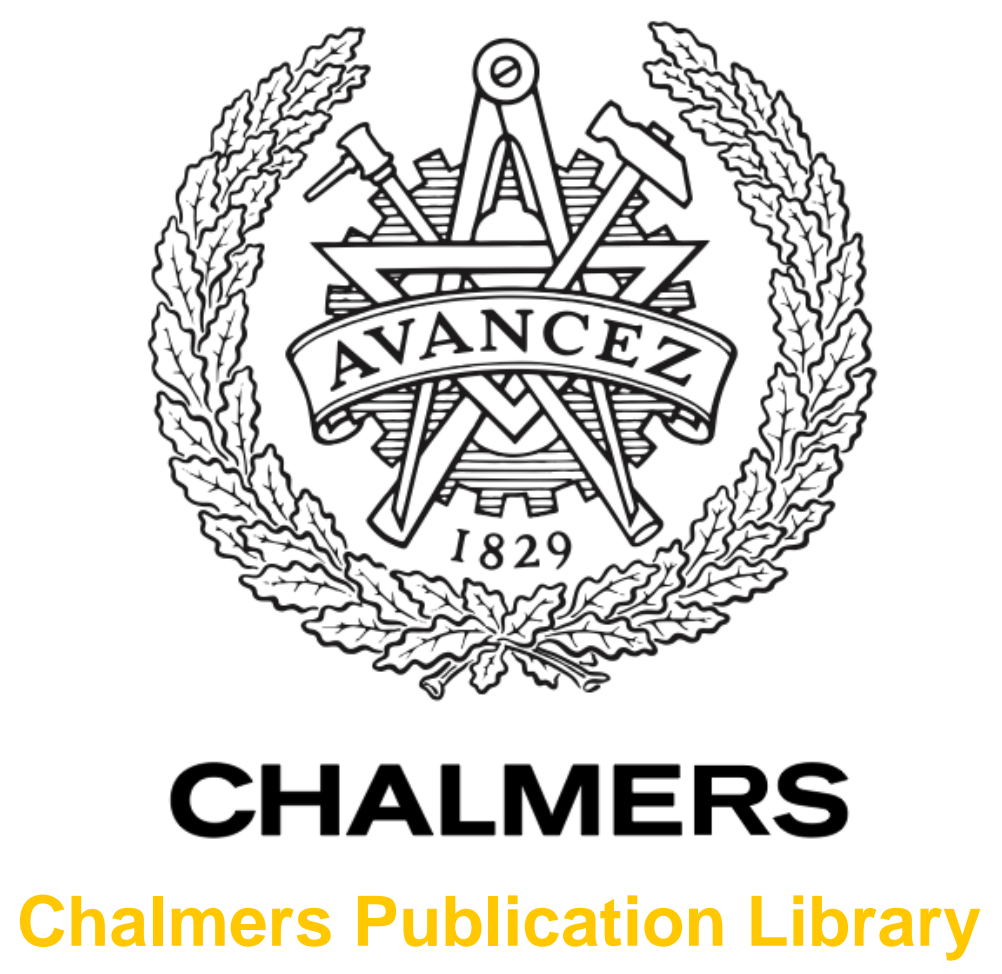

\title{
A strong magnetic field in the jet base of a supermassive black hole
}

This document has been downloaded from Chalmers Publication Library (CPL). It is the author's version of a work that was accepted for publication in:

Science (ISSN: 0036-8075)

Citation for the published paper:

Marti-Vidal, I. ; Muller, S. ; Vlemmings, W. et al. (2015) "A strong magnetic field in the jet base of a supermassive black hole". Science, vol. 348(6232), pp. 311-314.

http://dx.doi.org/10.1126/science.aaa1784

Downloaded from: http://publications.lib.chalmers.se/publication/218253

Notice: Changes introduced as a result of publishing processes such as copy-editing and formatting may not be reflected in this document. For a definitive version of this work, please refer to the published source. Please note that access to the published version might require a subscription.

Chalmers Publication Library (CPL) offers the possibility of retrieving research publications produced at Chalmers University of Technology. It covers all types of publications: articles, dissertations, licentiate theses, masters theses, conference papers, reports etc. Since 2006 it is the official tool for Chalmers official publication statistics. To ensure that Chalmers research results are disseminated as widely as possible, an Open Access Policy has been adopted.

The CPL service is administrated and maintained by Chalmers Library. 


\section{REFERENCES AND NOTES}

1. G. Binnig, C. F. Quate, C. Gerber, Phys. Rev. Lett. 56, 930-933 (1986).

2. F. J. Giessibl, S. Hembacher, H. Bielefeldt, J. Mannhart, Science 289, 422-425 (2000)

3. H. J. Hug et al., Science 291, 2509a (2001).

4. F. J. Giessibl, H. Bielefeldt, S. Hembacher, J. Mannhart, Ann. Phys. 10, 887-910 (2001).

5. M. Herz, F. J. Giessibl, J. Mannhart, Phys. Rev. B 68, 045301 (2003).

6. A. N. Chaika, A. N. Myagkov, J. Phys. Conf. Ser. 100, 012020 (2008).

7. S. Hembacher, F. J. Giessibl, J. Mannhart, Science 305 380-383 (2004).

8. J. Welker, F. J. Giessibl, Science 336, 444-449 (2012).

9. T. Hofmann, F. Pielmeier, F. J. Giessibl, Phys. Rev. Lett. 112 066101 (2014)

10. M. Ternes, C. P. Lutz, C. F. Hirjibehedin, F. J. Giessibl, A. J. Heinrich, Science 319, 1066-1069 (2008).

11. A. Zangwill, Physics on Surfaces (Cambridge Univ. Press, Cambridge 1988)

12. Submolecular resolution is defined as the ability to resolve atomic positions within molecules. Here, we refer to subatomic resolution as the ability to image single atoms as nontrivial features such as tori or multiple extrema, in contrast to single protrusions or single depressions.

13. L. Gross, F. Mohn, N. Moll, P. Liljeroth, G. Meyer, Science 325 1110-1114 (2009).

14. L. Bartels, G. Meyer, K. H. Rieder, Appl. Phys. Lett. 71, 213 (1997).

15. L. Gross et al., Science 337, 1326-1329 (2012)

16. D. G. de Oteyza et al., Science 340, 1434-1437 (2013).

17. N. Moll, L. Gross, F. Mohn, A. Curioni, G. Meyer, New J. Phys. 12, 125020 (2010).

18. R. G. Gordon, Y. S. Kim, J. Chem. Phys. 56, 3122 (1972).

19. A. J. Weymouth, T. Hofmann, F. J. Giessibl, Science 343 1120-1122 (2014)

20. J. C. Slater, Phys. Rev. 36, 57-64 (1930).

21. Supplementary materials are available on Science Online.

22. R. Smoluchowski, Phys. Rev. 60, 661-674 (1941).

23. M. Schneiderbauer, M. Emmrich, A. J. Weymouth, F. J. Giessibl, Phys. Rev. Lett. 112, 166102 (2014)

24. M. Feng et al., ACS Nano 5, 8877-8883 (2011).

25. P. Hapala, R. Temirov, F. S. Tautz, P. Jelínek, Phys. Rev. Lett. 113, 226101 (2014).

26. G. Kresse, J. Hafner, Phys. Rev. B 47, 558-561 (1993).

27. M. Huang, M. Čuma, F. Liu, Phys. Rev. Lett. 90, 256101 (2003).

28. L. A. Zotti, W. A. Hofer, F. J. Giessibl, Chem. Phys. Lett. 420 , 177-182 (2006)

29. C. A. Wright, S. D. Solares, Nano Lett. 11, 5026-5033 (2011).

30. H. Brune, J. Wintterlin, G. Ertl, R. J. Behm, Europhys. Lett. 13 123-128 (1990)

31. J. Repp, G. Meyer, K.-H. Rieder, P. Hyldgaard, Phys. Rev. Lett. 91, 206102 (2003).

32. D. M. Eigler, E. K. Schweizer, Nature 344, 524-526 (1990).

33. J. A. Stroscio, R. J. Celotta, Science 306, 242-247 (2004).

34. M. F. Crommie, C. P. Lutz, D. M. Eigler, Science 262, 218-220 (1993).

35. A. Biedermann, W. Rupp, M. Schmid, P. Varga, Phys. Rev. B 73 165418 (2006).

36. M. Pivetta, G. E. Pacchioni, U. Schlickum, J. V. Barth, H. Brune, Phys. Rev. Lett. 110, 086102 (2013).

37. A. A. Khajetoorians et al., Science 339, 55-59 (2013).

38. B. Schuler et al., Phys. Rev. Lett. 111, 106103 (2013).

39. F. Pielmeier, F. J. Giessibl, Phys. Rev. Lett. 110, 266101 (2013).

40. Our initial experiments of evaporating Fe atoms on $\mathrm{Cu}(111)$ appeared to confirm the findings of the inverse of the threefold tip 3 of (8), because we found many threefold structures that we first misinterpreted as single atoms. It turned out that upon extended Fe evaporation, the copper crystal heated up to $\sim 15 \mathrm{~K}$ and the Fe monomers became mobile enough to form an abundance of trimers.

\section{ACKNOWLEDGMENTS}

We thank G. Binnig, J. Repp, and A. J. Weymouth for discussions and the Deutsche Forschungsgemeinschaft for funding under Graduiertenkolleg 1570 and Sonderforschungsbereich 689, as well as M. Grifoni (speaker of GRK) and D. Weiss (speaker of SFB) for support. F.J.G. thanks A. Heinrich for hosting several visits at the IBM Almaden Research Center from 2005 until 2010, where first attempts to resolve metallic adatoms with subatomic resolution were performed together with C. Lutz, C. Hirjibehedin, and M. Ternes. Author contributions: M.E. performed most of the measurements and data analysis, constructed the low-temperature scan head, and prepared most figures; F.H. optimized the electronics and performed crucial measurements; J.W. performed measurements on $\mathrm{Cu}$ adatoms on $\mathrm{Cu}(111) ; \mathrm{T} . \mathrm{H}$. and F.P. performed measurements on $\mathrm{Cu} / \mathrm{Cu}(110)$ and inverse measurements using
$\mathrm{CO} / \mathrm{Cu}(111)$ to characterize $\mathrm{Cu}$ and Fe tips; M.S. constructed most of the vacuum system and sample-preparation facilities; D.M. performed the AFM measurements on Si; D.K. S.P., S.M., and H.E. performed the DFT calculations; and F.J.G. initiated and directed the project, employed the STO model, and wrote the manuscript.

\section{SUPPLEMENTARY MATERIALS}

www.sciencemag.org/content/348/6232/308/suppl/DC1 Materials and Methods

Supplementary Text

Figs. S1 to S13

References (41-47)

19 December 2014; accepted 20 February 2015

Published online 19 March 2015:

10.1126/science.aaa5329

\section{A strong magnetic field in the jet base of a supermassive black hole}

\author{
Ivan Martí-Vidal, ${ }^{*}$ Sébastien Muller, Wouter Vlemmings, Cathy Horellou, Susanne Aalto
}

Active galactic nuclei (AGN) host some of the most energetic phenomena in the universe. AGN are thought to be powered by accretion of matter onto a rotating disk that surrounds a supermassive black hole. Jet streams can be boosted in energy near the event horizon of the black hole and then flow outward along the rotation axis of the disk. The mechanism that forms such a jet and guides it over scales from a few light-days up to millions of light-years remains uncertain, but magnetic fields are thought to play a critical role. Using the Atacama Large Millimeter/submillimeter Array (ALMA), we have detected a polarization signal (Faraday rotation) related to the strong magnetic field at the jet base of a distant AGN, PKS 1830-211. The amount of Faraday rotation (rotation measure) is proportional to the integral of the magnetic field strength along the line of sight times the density of electrons. The high rotation measures derived suggest magnetic fields of at least tens of Gauss (and possibly considerably higher) on scales of the order of light-days ( 0.01 parsec) from the black hole.

$\mathrm{I}$ he AGN jets, related to the accretion mechanism in supermassive black holes, consist of relativistic plasma driven by strong and ordered magnetic fields. As a result of the magnetic interaction of the plasma, nonthermal (synchrotron) emission is produced (I). Studying the polarization of this nonthermal emission from AGN is a direct way to probe the structure and strength of magnetic fields in the vicinity of a black hole. Of particular importance is the observation of the rotation measure, $R M$, defined as the change of polarization angle as a function of wavelength squared. This quantity is directly related to the plasma density and the strength of the magnetic field along the line of sight.

To date, it has been extremely difficult to obtain accurate polarimetric information from the innermost (subparsec) regions of AGN; only emission at submillimeter wavelengths can escape from these regions, due to a large synchrotron self-absorption (SSA) that blocks the emission

Department of Earth and Space Sciences, Chalmers

University of Technology, Onsala Space Observatory, SE-43992, Onsala, Sweden.

*Corresponding author. E-mail: mivan@chalmers.se at longer wavelengths. Unfortunately, the sensitivity of polarization observations at submillimeter wavelengths has so far been strongly limited by the instrumentation.

Previous attempts to detect Faraday rotation at submillimeter (submm) wavelengths from AGN have yielded only upper limits $(2,3)$ and marginal detections (4) that require strong assumptions about the absence of variability on time scales of weeks. There is a more robust detection for the Galactic center (5), although the activity in this source is much lower than in AGN. Recently, measurements of Faraday rotation in the nearby AGN 3C 84 (redshift $z=0.018$ ) have been reported at $\mathrm{mm}$ wavelengths (6).

We have obtained measurements of Faraday rotation at frequencies up to $300 \mathrm{GHz}$ (about 1830-211, a powerful gravitationally lensed AGN located at a redshift of $z=2.5$ (7). At these frequencies, SSA is negligible in the whole jet of PKS 1830-211 (8), and the maximum emission originates at the jet region closest to the black hole; the zone where the plasma is being injected and accelerated into the main jet stream. At lower frequencies, SSA hides this jet acceleration zone $1 \mathrm{THz}$ in the rest frame of the source) from PKS 
from view. These results are thus fundamental to better understand the role of magnetic fields in the AGN accretion and jet production, which are intimately related to the growth and evolution of supermassive black holes.

This detection has been possible thanks to the high resolution (sub-arcsec) of our observations with ALMA and to the use of a new differential polarimetry technique, which we briefly describe in the following lines (see supplementary text section 1).

The ALMA receivers detect the signal in two orthogonal linear polarizations, $\mathrm{X}$ and $\mathrm{Y}$, where $\mathrm{X}$ is received from a horizontal dipole and $\mathrm{Y}$ from a vertical dipole in the frame of the antenna mount. The two lensed images of PKS 1830-211, which we call northeast (NE, upper-left in projection on sky) and southwest (SW, lower- right), are separated by $1^{\prime \prime}$. In Fig. 1, we show an example of snapshot images in XX and YY of the two components of the gravitational lens, as well as their difference. The difference image contains information about the difference between $\mathrm{NE}$ and SW in Stokes parameters $Q$ and $U$. Our analysis makes use of the polarization ratio, $R_{\text {pol }}$, which is defined as

$$
R_{\mathrm{pol}}=\frac{1}{2}\left(\frac{R_{\mathrm{XX}}^{1,2}}{R_{\mathrm{YY}}^{1,2}}-1\right)
$$

where $R_{\mathrm{XX}}^{1,2}$ and $R_{\mathrm{YY}}^{1,2}$ are the flux-density ratios between the two lensed images of the AGN, obtained separately from the XX and YY polarization products. $R_{\mathrm{pol}}$ is a function of the parallactic angle of the antennas, $\psi$, and the observing wavelength, $\lambda$, and encodes information about the difference of polarization between the two images, via the approximately constant parameters $p_{\text {dif }}$ and $\alpha$ (supplementary text section 1$)$, as well as their rotation measure $R M$,

$$
R_{\mathrm{pol}}=p_{\text {dif }} \cos \left(2{\phi^{\prime}}_{1}^{0}-\alpha+2 R M\left(\lambda^{2}-\psi / R M\right)\right)
$$

where $\phi_{1}^{\prime}$ is the position angle of the polarization of image 1 at zero wavelength in the plane of the sky. The technique of differential polarimetry essentially enables estimation of $R M$ via fitting the observed sinusoidal dependence of $R_{\text {pol }}$ as a function of $\lambda^{2}$ and $\psi$, using Eq. 1 .

Our results are based on ALMA observations at sky frequencies around 100, 250, and $300 \mathrm{GHz}$ (8). Correcting for the cosmological redshift, these frequencies correspond to 350,875 , and $1050 \mathrm{GHz}$
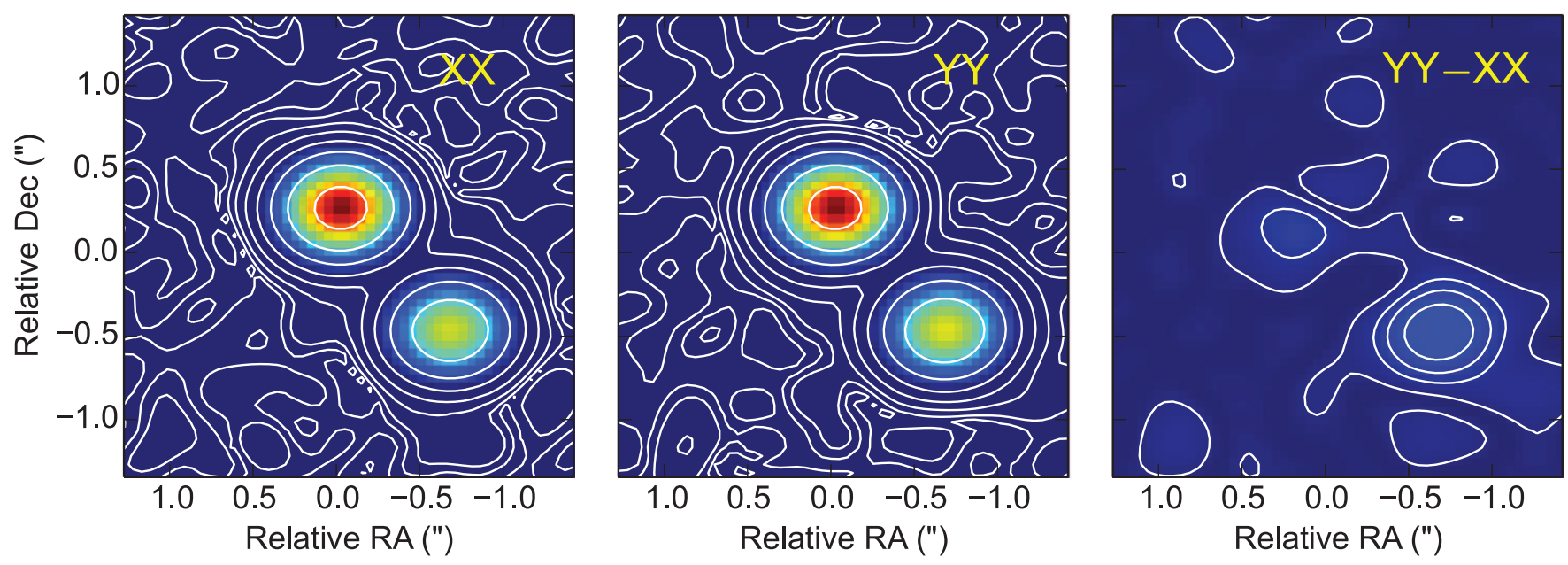

Fig. 1. ALMA image of the gravitationally lensed AGN PKS 1830-211 at 250 GHz, taken on 30 June 2014. Left, in XX polarization; center, in YY polarization (with the peak normalized to that of the $X X$ image); right, the difference between polarizations. Notice the small residual in the southwest lensed image, which encodes differential polarization information among the northeast and southwest images. The contours are set at $0.625,1.25,2.5,5$, $10,20,50$, and $99 \%$ of the peak intensity.
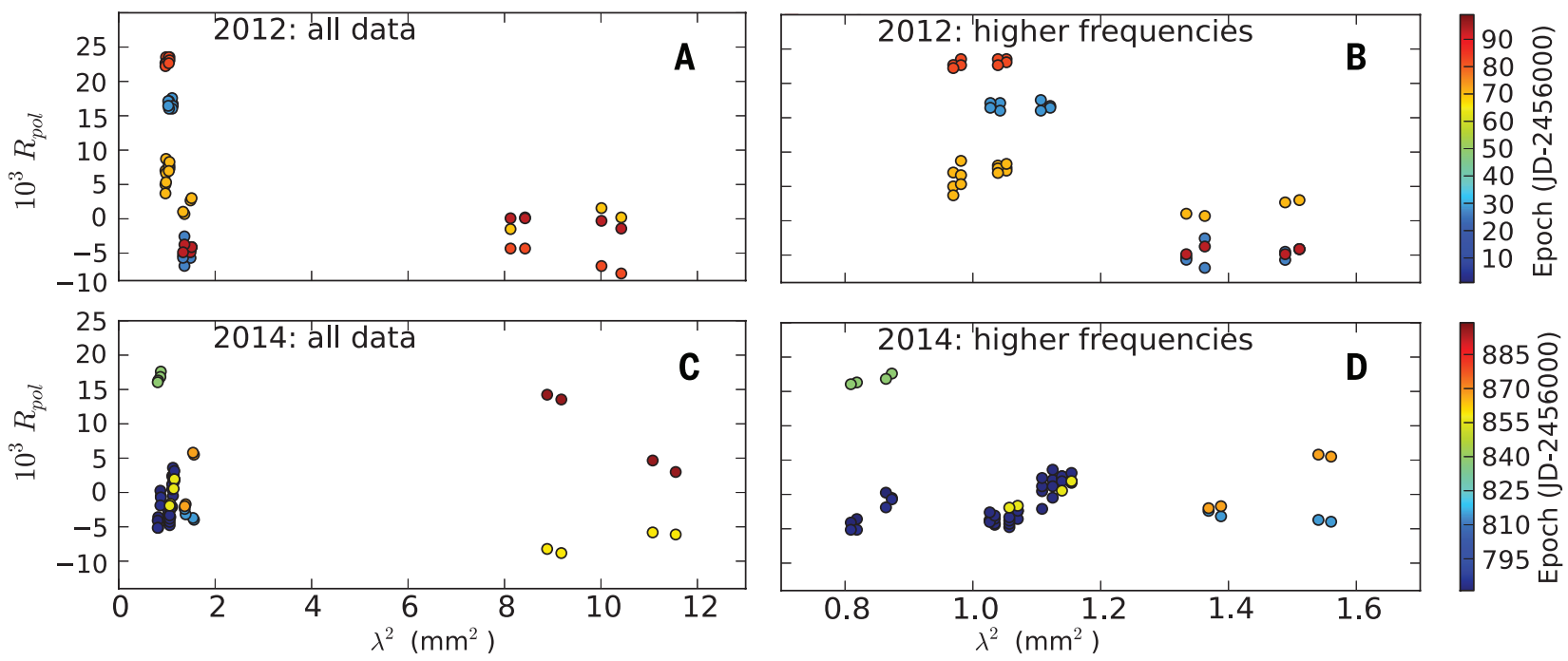

Fig. 2. Polarization ratio, $\boldsymbol{R}_{\text {pol, }}$ as a function of the wavelength squared for all our ALMA observations. Left panels (A and $\mathbf{C}$ ) are all data; right panels (B and $\mathbf{D}$ ) are enlargements of the region of shorter wavelengths. The uncertainties, estimated from the postfit covariance matrix as described in (10), are of the order of the symbol sizes. 
in the frame of the source. More details on these observations, and a summary of the main goals of this ALMA project, can be found elsewhere (9). We also summarize all the observations in the supplementary text (section 2). Our observations can be divided in two data sets, one consisting of other of nine epochs in 2014 (3 May to 27 August). In Fig. 2, we show the measured $R_{\text {pol }}$ between the two lensed images of PKS 1830-211. These measurements have been obtained from the $R_{\mathrm{XX}}$ and $R_{\mathrm{YY}}$ values fitted with the visibility-modeling software presented in (10). The uncertainties have been obtained with the standard error propagation approach, using the uncertainties in $R_{\mathrm{XX}}$ and $R_{\mathrm{YY}}$ that were derived from the cosix epochs in 2012 (9 April to 16 June) and the

variance matrix of the visibility fitting, as described in (10).

The derivatives of $R_{\mathrm{pol}}$ versus $\lambda^{2}$, which are related to $R M$ (supplementary text section 1.3), are clearly different for different wavelength ranges. Between $\lambda^{2}=8$ and $12 \mathrm{~mm}^{2}$, the maximum derivative is $4.4 \times 10^{-3} \mathrm{~mm}^{-2}$, whereas between 0.8 and $1.6 \mathrm{~mm}^{2}$ it is $70 \times 10^{-3} \mathrm{~mm}^{-2}$. Because the maximum observed $R_{\mathrm{pol}}$ ratios are, in absolute value, similar at all wavelengths, the different derivatives of $R_{\mathrm{pol}}$ versus $\lambda^{2}$ must be due to larger $R M$ at shorter wavelengths (see supplementary text section 1.3 for a more detailed discussion). Large variations of $R M$ with wavelength have been reported in other AGN (11), although at much longer wavelengths $(\mathrm{cm})$, related

\section{Fig. 3. Sketch of the jet launch/acceleration region in PKS 1830-211 (not to} scale). Emission at higher frequencies comes from material closer to the black hole, at subparsec scales. At these frequencies, the main contribution to RM must come from a zone close to the jet, in order to explain the different $R M$ values between $350 \mathrm{GHz}$ and 0.8 to $1 \mathrm{THz}$ (source frame).

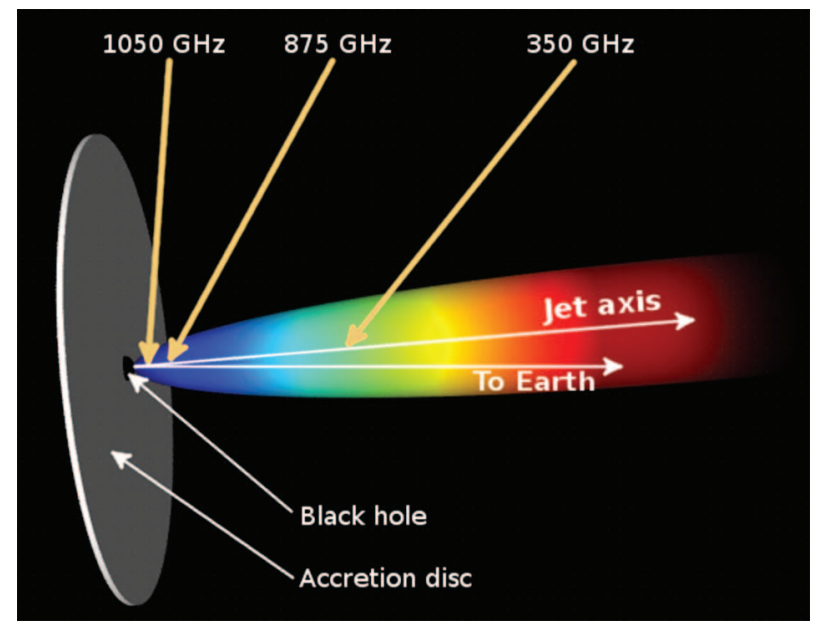

Fig. 4. Fits of our three epochs with quasisimultaneous observations at 250 and $300 \mathrm{GHz}$ to the model given in Eq. 1. We show $R_{\text {pol }}$ versus $\lambda^{2}$ corrected by $-\psi / R M$, to obtain a sinusoidal behavior.

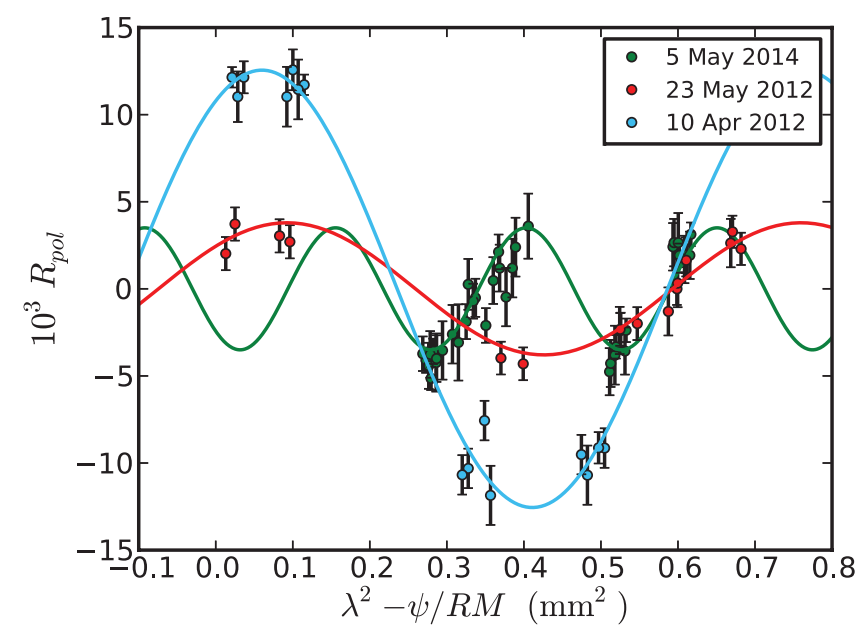

Table 1. Best-fit polarization values for the three epochs with quasi-simultaneous observations at 250 and $300 \mathrm{GHz}$. $R M_{\mathrm{obs}}$ are the rotation measures in the observer's frame and $R M_{\text {true }}$ are the rotation measures in the rest frame of the source. $R M_{\text {true }}$ is $(1+z)^{2}$ times larger than $R M_{\text {obs }}$.

\section{Epoch}

\begin{tabular}{lccc} 
& 10 April 2012 & 23 May 2012 & 5 May 2014 \\
\hline$R M_{\text {obs }}\left(10^{6} \mathrm{rad} / \mathrm{m}^{2}\right)$ & $9.0 \pm 0.3$ & $9.4 \pm 0.4$ & $25.3 \pm 0.8$ \\
$R M_{\text {true }}\left(10^{7} \mathrm{rad} / \mathrm{m}^{2}\right)$ & $11 \pm 0.4$ & $11.5 \pm 0.5$ & $31.2 \pm 1.0$ \\
$p_{\text {dif }}\left(10^{-3}\right)$ & $12.6 \pm 0.4$ & $3.8 \pm 0.3$ & $3.5 \pm 0.3$ \\
$2 \phi_{\mathrm{o}}-\alpha(\mathrm{deg})$ & $59 \pm 27$ & $40 \pm 23$ & $25 \pm 20$ \\
\hline
\end{tabular}

to larger spatial scales in the jets. Our finding cannot be explained easily if the $R M$ is only caused by an external (e.g., spherically symmetric) screen of material being accreted onto the black hole [as in the case of the $R M$ detected in the Galactic center (5)] and/or by external clouds. The size of the submm emitting region (estimated as the distance to the black hole at which the submm intensity is maximum) is only of the order of $0.01 \mathrm{pc}(8)$. Hence, if the Faraday screen were extended and located far from the jet base, the rotation measure at submm wavelengths should not depend on the observing frequency, because the extent of the Faraday screen would be similar for all the submm jet emission. The Faraday screen must thus be close to the jet base and change substantially on sub-parsec scales (Fig. 3). An increase of the $R M$ at shorter wavelengths would then be explained naturally as an increase of the magnetic field strength and/or electron density as we approach the black hole. Indeed, observations of other AGN at long wavelengths (cm) show changes of $R M$ across the jets, both longitudinal and transversal (12-14), that have been attributed to changes in particle density and magnetic fields in the jets, independent of a more distant external medium.

We have three sets of observing epochs at 250 and $300 \mathrm{GHz}$ separated by a short time interval (1 to 2 days). In these three cases, we can directly estimate $R M$ and $p_{\text {dif }}$ by fitting $R_{\text {pol }}$ to the model given by Eq. 1 . The parameter estimates in these three data sets have been performed by leastsquares minimization, comparing the measured $R_{\text {pol }}$ to the model predictions. The data at our lowest-frequency band (i.e., $100 \mathrm{GHz}$ ) have been discarded from the fit, because they trace different rotation measures from different regions of the jet, as we have already discussed. We show the fitting results in Fig. 4 and the estimated parameters in Table 1. Our estimated source-rest-frame $R M \mathrm{~s}$ are about two orders of magnitude higher than the highest values reported previously for other AGN, which are $\sim 10^{6} \mathrm{rad} / \mathrm{m}^{2}(4,6)$.

Although the two $R M$ measurements in 2012 are compatible, the estimate in 2014 is higher by more than a factor of 2 . Regarding the amplitude of $R_{\text {pol }}$, which is related to the fractional polarization and to the relative polarization angles among the NE and SW images, we find different values for the two observations in 2012. These two observations were serendipitously taken before and after a strong $\gamma$-ray flare, which had a very weak radio counterpart (8). This leads us to speculate that the change in polarization may be correlated to the radio counterpart of that flare. Another $\gamma$-ray flare was detected in 2014 (15), also coincident with the time range of our 2014 observations. The new flare had a strong radio counterpart, which may also be related to the higher $R M$ that we measure in 2014. The high variability in $R M$ and $p_{\text {dif }}$, in connection to the $\gamma$-ray flaring events, points toward a cospatial origin of the $\gamma$-ray emission and the 250- to $300-\mathrm{GHz}$ rotation measures, hence favoring our interpretation of the $R M$ being caused at the region very close to the jet base. 
The $R M$ is related to the line-of-sight integral of the electron density times the magnetic field, corrected for the cosmological redshift (16). In units of $\mathrm{rad} / \mathrm{m}^{2}$,

$$
R M=8.1 \times 10^{5} \frac{1}{(1+z)^{2}} \int_{n} B_{\|} d l
$$

where $d l$ is the differential path along the line of sight (in pc), $z$ is the redshift ( $z=2.5$ ), $n$ is the particle density (in $\mathrm{cm}^{-3}$ ), and $B_{\|}$is the magnetic field projected in the line of sight (in Gauss). Our $R M$ will be an important test for detailed magnetohydrodynamical (MHD) models at the jet base, but such analysis is beyond the scope of this paper. At a more basic level, it is impossible to unambiguously disentangle the contributions of the magnetic field, electron density, and path length to the integral determining the rotation measure. This difficulty is exacerbated by the absence of direct information about the electron density or path length from observations, leading to the need to extrapolate from larger scales, which introduces additional uncertainty. The rotation measures derived here, $R M \sim 10^{8} \mathrm{rad} / \mathrm{m}^{2}$ in the rest frame of the source, are about a factor of $10^{5}$ greater than the rest-frame $R M$ values measured for parsec-scale AGN cores, where the derived magnetic fields have been independently measured to be $\sim 0.05$ to $0.10 \mathrm{G}(11,17)$; this suggests that the magnetic fields in the subparsec regions that we are probing are at least a few tens of Gauss, and possibly much higher. More exact estimates of these magnetic fields will require a separate dedicated study.

In any case, our results are a clear indication of very high magnetic fields at the jet base, which should be dynamically important near the black hole and should in turn affect the accretion process. A similar conclusion was drawn from a statistical analysis of jet core shifts from a complete sample of AGN, using high-resolution radio observations at centimeter wavelengths (18). In the near future, our differential polarimetry technique can be used to further measure and monitor $R M \mathrm{~s}$ at very short wavelengths, from this and other AGN. The monitoring of magnetic fields and particle densities at the jet regions closest to the black holes, via submm polarimetry, will allow us to study the tight connection between black-hole accretion and relativistic jets, the two fundamental pieces of the fascinating cosmic puzzle of AGN.

\section{REFERENCES AND NOTES}

1. A. P. Marscher, Astrophys. J. 235, 386 (1980).

2. I. Agudo, C. Thum, J. L. Gómez, H. Wiesemeyer, Astron. Astrophys. 566, A59 (2014)

3. C. Y. Kuo, K. Asada, R. Rao, M. Nakamura, J. C. Algaba, H. B. Liu, M. Inoue, P. M. Koch, P. T. P. Ho, S. Matsushita, H.-Y. Pu, K. Akiyama, H. Nishioka, N. Pradel, Astron. Astrophys. 783, 33 (2014)

4. S. Trippe et al., Astron. Astrophys. 540, A74 (2012).

5. D. P. Marrone, J. M. Moran, J.-H. Zhao, R. Rao, Astrophys. J. 654, L57-L60 (2007)

6. R. L. Plambeck et al., Astrophys. J. 797, 66 (2014).

7. C. Lidman et al., Astrophys. J. 514, L57-L60 (1999).

8. I. Martí-Vidal et al., Astron. Astrophys. 558, A123 (2013).

9. S. Muller et al., Astron. Astrophys. 566, A112 (2014).
10. I. Martí-Vidal, W. Vlemmings, S. Muller, S. Casey, Astron. Astrophys. 563, A136 (2014).

11. S. P. O'Sullivan, D. C. Gabuzda, Mon. Not. R. Astron. Soc. 393 , 429-456 (2009)

12. S. M. Croke, S. P. O'Sullivan, D. C. Gabuzda, Mon. Not. R. Astron. Soc. 402, 259-270 (2010).

13. M. Mahmud, D. Gabuzda, V. Bezrukovs, Mon. Not. R. Astron. Soc. 400, 2-12 (2009).

14. D. C. Gabuzda, V. A. Chernetskii, Mon. Not. R. Astron. Soc. 339 , 669-679 (2003).

15. S. Ciprini, The Astronomers Telegram 4158 (2012).

16. M. L. Bernet, F. Miniati, S. Lilly, Astrophys. J. 761, 144 (2012)

17. S. P. O'Sullivan, D. C. Gabuzda, Mon. Not. R. Astron. Soc. 400 26-42 (2009)

18. M. Zamaninasab, E. Clausen-Brown, T. Savolainen, A. Tchekhovskoy, Nature 510, 126-128 (2014).

\section{ACKNOWLEDGMENTS}

This paper makes use of the following ALMA data: ADS/JAO. ALMA\#2011.0.00405.S, ADS/JAO.ALMA\#2012.1.00056.S, and
ADS/JAO.ALMA\#2013.1.00020.S. ALMA is a partnership of the European Southern Observatory (ESO) (representing its member states), U.S. National Science Foundation (NSF), and National Institutes of Natural Sciences (NINS) of Japan, together with the National Research Council of Canada (NRC) and National Science Council of Taiwan (NSC) and Academia Sinica Institute of Astronomy and Astrophysics (ASIAA) (Taiwan), in cooperation with the Republic of Chile. The Joint ALMA Observatory is operated by ESO, Associated Universities, Inc.-National Radio Astronomy Observatory, and National Astronomical Observatory of Japan. W.V. acknowledges support from the European Research Council (ERC) under ERC grant 614264.

\section{SUPPLEMENTARY MATERIALS}

www.sciencemag.org/content/348/6232/311/suppl/DC1 Supplementary Text

Figs. S1 to S4

Tables S1 to S3

Reference (19)

28 October 2014; accepted 12 March 2015

10.1126/science.aaa1784

\title{
GALAXY EVOLUTION
}

\section{Evidence for mature bulges and an inside-out quenching phase 3 billion years after the Big Bang}

\author{
S. Tacchella, ${ }^{1 *}$ C. M. Carollo, ${ }^{1 *}$ A. Renzini, ${ }^{2}$ N. M. Förster Schreiber, ${ }^{3}$ P. Lang, ${ }^{3}$ \\ S. Wuyts, ${ }^{3}$ G. Cresci, ${ }^{4}$ A. Dekel, ${ }^{5}$ R. Genzel,${ }^{3,6,7}$ S. J. Lilly, ${ }^{1}$ C. Mancini, ${ }^{2}$ S. Newman, ${ }^{6}$ \\ M. Onodera, ${ }^{1}$ A. Shapley, ${ }^{8}$ L. Tacconi, ${ }^{3}$ J. Woo, ${ }^{1}$ G. Zamorani ${ }^{9}$
}

Most present-day galaxies with stellar masses $\geq 10^{11}$ solar masses show no ongoing star formation and are dense spheroids. Ten billion years ago, similarly massive galaxies were typically forming stars at rates of hundreds solar masses per year. It is debated how star formation ceased, on which time scales, and how this "quenching" relates to the emergence of dense spheroids. We measured stellar mass and star-formation rate surface density distributions in star-forming galaxies at redshift 2.2 with $\sim 1$-kiloparsec resolution. We find that, in the most massive galaxies, star formation is quenched from the inside out, on time scales less than 1 billion years in the inner regions, up to a few billion years in the outer disks. These galaxies sustain high star-formation activity at large radii, while hosting fully grown and already quenched bulges in their cores.

A t the epoch when star-formation activity peaks in the universe [redshift $z \sim 2(1,2)]$, massive galaxies typically lie on the socalled "star-forming main sequence." Their star-formation rates (SFRs) tightly correlate with the mass in stars (stellar mass $M$ ), reaching up to several hundred solar masses $\left(M_{\odot}\right)$ per

${ }^{1}$ Department of Physics, Institute for Astronomy, ETH Zurich, CH-8093 Zurich, Switzerland. ${ }^{2}$ Istituto Nazionale di Astrofisica (INAF) Osservatorio Astronomico di Padova, Vicolo dell Osservatorio 5, I-35122 Padova, Italy. ${ }^{3}$ Max-Planck-Institut für Extraterrestrische Physik, Giessenbachstrasse 1, D-85748 Garching, Germany. ${ }^{4}$ INAF Osservatorio Astronomico di Arcetri, Largo Enrico Fermi 5, I-50125 Firenze, Italy. ${ }^{5}$ Racah Institute of Physics, The Hebrew University, Jerusalem 91904, Israel. ${ }^{6}$ Department of Astronomy, Campbell Hall, University of California, Berkeley, CA 94720, USA. ${ }^{7}$ Department of Physics, Le Conte Hall, University of California, Berkeley, CA 94720, USA. ${ }^{8}$ Department of Physics and Astronomy, University of California, Los Angeles, CA 90095-1547, USA. ${ }^{9}$ INAF Osservatorio Astronomico di Bologna, Via Ranzani 1, I-40127 Bologna, Italy.

${ }^{*}$ Corresponding author. E-mail: sandro.tacchella@phys.ethz.ch (S.T.); marcella.carollo@phys.ethz.ch (C.M.C.) year and producing a characteristic specific SFR $(\mathrm{SSFR}=\mathrm{SFR} / M)$ that declines only weakly with mass $(3,4)$. In contrast, at the present epoch, such massive galaxies are spheroids with old stellar populations, which reach central surface stellar densities well above $10^{10} M_{\odot} \mathrm{kpc}^{-2}$ and host virtually no ongoing star formation. Although the most massive ellipticals at $z=0$ bear the clear signatures of a gas-poor formation process $(5,6)$, the more typical population, at a mass scale of $M \sim 10^{11} M_{\odot}$, consists of fast rotators (7) with disklike isophotes (8), steep nuclear light profiles (9), and steep metallicity gradients (10): all features that indicate a gas-rich formation process.

The full cessation of star-formation activity in these typical massive galaxies (here referred to as the quenching process) is not well understood, nor is its relation with the emergence of their spheroidal morphologies. Several quenching mechanisms have been proposed. The so-called haloquenching scenario predicts that circumgalactic gas is shock-heated to high temperatures and 
If you wish to distribute this article to others, you can order high-quality copies for your colleagues, clients, or customers by clicking here.

Permission to republish or repurpose articles or portions of articles can be obtained by following the guidelines here.

The following resources related to this article are available online at www.sciencemag.org (this information is current as of November 13, 2015 ):

Updated information and services, including high-resolution figures, can be found in the online version of this article at:

http://www.sciencemag.org/content/348/6232/311.full.html

Supporting Online Material can be found at:

http://www.sciencemag.org/content/suppl/2015/04/15/348.6232.311.DC1.html

This article cites 18 articles, 5 of which can be accessed free:

http://www.sciencemag.org/content/348/6232/311.full.html\#ref-list-1

This article appears in the following subject collections:

Astronomy

http://www.sciencemag.org/cgi/collection/astronomy 